\title{
QUANTUM AND NANOSCALE MODELLING OF EXCITON DYNAMICS IN POLYMERIC SYSTEMS
}

\author{
H. M. C. Barbosa ${ }^{*}$, H.M.G. Correia and M. M. D. Ramos. \\ Research Centre and Department of Physics, University of Minho \\ Campus de Gualtar, 4710-057 Braga, Portugal \\ Tel.: +351 253604 330; Fax: +351 253604061 \\ helder@fisica.uminho.pt (H. M. C. Barbosa)
}

*Corresponding author

\begin{abstract}
One of the factors that limit the efficiency of polymer-based optoelectronic devices, such as photovoltaic solar cells and light emitting diodes, is the exciton diffusion within the polymeric network. Due to the amorphous nature the of polymeric materials, the diffusion of excitons is limited by the energetic and spatial disorder in such systems, which is a consequence not only of the chemical structure of the polymer but also from its morphology at nanoscale.

To get a deep understanding on how such effects influence exciton dynamics we performed a quantum molecular dynamics simulations to determine the energetic disorder within the polymer system, and Monte Carlo simulations to study exciton diffusion in three-dimensional (3D) polymer networks that present both spatial and energetic disorder at nanometre scale. Our results show clearly that exciton diffusion in poly(pphenylenevenylene) (PPV) occurs preferentially in the direction parallel to the electrodes surface for a polymer-based optoelectronic devices with the orientation of the conjugated
\end{abstract}


strands similar to those obtained by the spin-coating technique and the decay of such excitons occurs preferentially in longer strands which allow us to get insight on exciton behaviour in polymeric systems that are not possible to be obtained directly from the experiments.

Keywords: Excitons, PPV, atomistic modelling, nanoscale modelling, diffusion length, diffusion coefficient.

\section{Introduction}

During the last years there has been intense studies on organic solar cells (OSC) based on blended low weight molecules with polymers, forming a bulk heterojunction ${ }^{1}$. In these devices, photons are absorbed by the polymeric material, leading to the generation of intramolecular electronic excited states known as excitons that diffuse through the polymer material until they decay take place. One of the conditions to increase photocurrent in this organic solar cell is to improve the probability of an exciton to reache a polymer/molecule interface within the bulk heterojunction. Efficient exciton dissociation (i.e. hole-electron separation) ${ }^{2}$ takes place due to the different electron affinities of the both materials. Although increasing the interface area between the two materials should improve charge separation, this strategy will not change significantly the photocurrent because it reduces drastically the free pathways for charge transport within the blend and charge collection by the electrodes. To maximize simultaneously exciton dissociation and charge transport towards the electrodes, several architectures have been proposed $^{1,3}$ for OSC in order to obtain an average distance between two polymer/molecule interfaces similar to the exciton diffusion length. 
In the solid state, conjugated polymer chains have kinks and twists disrupting the conjugation. Depending on the deposition conditions, an assemble of stiff-chain conjugated segments with different lengths and orientations relative to each other and relative to the electrode surface is created within the polymer, giving rise to an anisotropic system. When the interaction between the neighbour conjugated segments is weak, atomistic calculations have shown that each conjugated segment can behave like an independent chromophore, and the formation of a singlet exciton, due to light absorption, occurs at the centre of the chromophore leading to a dipole charge distribution along the conjugated polymer backbone ${ }^{4}$, which corresponds to a Frenkeltype exciton. These results suggest that at nanometre scale the exciton diffusion should be treated as a $3 \mathrm{D}$ process rather than a $1 \mathrm{D}$ process. Moreover, the atomistic calculations have also shown that exciton energy depends on the conjugated segment length ${ }^{5}$. Therefore, energetic disorder should occur within the 3D-polymer network which has consequences for exciton diffusion.

The theoretical study of exciton migration in polymeric systems can easily be achieved using a dynamical Monte Carlo method. Scheidler et al. ${ }^{6}$ were the first to report a theoretical study of exciton migration in conjugated polymer systems. Nevertheless, they did not consider the effect of the polymer morphology at nanometre scale on exciton diffusion. Assuming identical approach, Watkins et al. ${ }^{3}$ report the effect of phase separation on organic bulk heterojunction solar cells in the exciton motion towards donor/acceptor interfaces. However, they did not take into account the influence of the orientational disorder on the transition dipole moment. Recently Ahn et al. ${ }^{7}$ have shown the effect of such orientational disorder in 1D exciton migration, assuming a Forster 
mechanism. In all these works the energetic disorder is considered by assuming a Gaussian distribution of exciton energies in the polymer segments.

In order to get a better understanding of the influence of the energetic and spatial disorder on singlet exciton diffusion in polymeric systems we performed two-scale computational modelling using PPV as a model system: i) atomistic quantum molecular dynamics calculations to determine exciton energy on chromophores with different conjugation lengths (i.e. number of monomer units at relaxed geometry); ii) Monte Carlo simulations to study exciton dynamics on 3D-polymer networks that present spatial and energetic disorder at nanometre scale. The main aim of this work is to get a clear picture of the effects of energetic and spatial disorder on exciton migration within polymeric systems, which are of fundamental importance for the design and improvement of polymer-based solar cells as well as other polymer-based devices.

\section{Model and computer simulation details}

When the active component of OSC based on polymeric materials is deposited by spin-coating, the preferential orientation of the longer axis of the conjugated polymer

segments are parallel to the electrodes surface ${ }^{8}$. In order to mimic the spatial disorder in this nanostructure, we built a 3D network of straight PPV segments placing them randomly in the gap between two planar electrodes separated by $100 \mathrm{~nm}$ (typical thickness of an organic film in optoelectronic devices), with their long molecular axis parallel to the electrodes surface, a uniform random distribution of the short axis and a minimum distance between them of $0.650 \mathrm{~nm}^{9}$. The segments within the network have a Gaussian distribution of lengths with a mean value of 7 monomers, which is a typical value obtained for the average conjugation length of PPV. 
Due to its nature, the absorption of a photon by a semiconducting polymer, such as PPV, leads to the formation of an intramolecular singlet exciton which induces both charge distribution and lattice distortion within the chromophore and thus it must be treated self-consistently at molecular level ${ }^{4}$. Therefore, to obtain the energy of the lowest singlet excited state of PPV strands with different length which are present within the polymer network and, thus, the energy barrier that an exciton has to overcome to hop between two chromophores, we use a self-consistent quantum chemistry method combined with a molecular dynamics method ${ }^{10}$. The combination of these two methods allow us obtain simultaneously the electronic structure and atomic relaxation of the polymer chromophore when the exciton is formed. For more details of the quantum molecular dynamics method used in this work see Ref. 4.

After generating a pulse of excitons inside a polymer network, there is exciton migration which can be mediated by different processes corresponding to different microscopic mechanisms. The exciton migration model that we present here is based on the Forster formalism, and the microscopic mechanism for exciton hopping between neighbouring polymer segments has a dependence on the energetic and spatial (i.e. distance and orientation between segments) disorder.

Each exciton created on a conjugated segment has an intrinsic lifetime given by:

$$
t_{i}=-\frac{\ln (X)}{w_{0}}
$$

where $X$ is a random number between 0 and 1 , and $w_{0}$ represents the decay probability of the excited state per unit time by spontaneous photon emission (radiative decay rate). Since we do not consider the existence of nonradiative decay channels inside the PPV 
network, a radiative decay rate of $1.25 \times 10^{9} \mathrm{~s}^{-1}$ expected for pristine PPV (see Ref 12) is used in this work.

The exciton hopping rate between two neighbouring polymer segments is given by:

$$
w_{i j}=w_{0, d i p-d i p} \times\left\{\begin{array}{ll}
1 \quad \text { for } r_{i j}<R \\
\left(\frac{R}{r_{i j}}\right)^{6} \text { for } r_{i j} \geq R
\end{array} \times \begin{cases}1 & \text { for } \Delta E_{i j} \leq 0 \\
\exp \left(-\frac{\Delta E_{i j}}{k T}\right) & \text { for } \Delta E_{i j}>0\end{cases}\right.
$$

The first term $w_{0, \text { dip-dip }}$, represents the attempt-to-escape frequency of an exciton, which is assumed to have the value of $3.3 \times 10^{-11} \mathrm{~s}^{-16}$. The second term falls off with the distance between the centres of the two polymer segments involved, $r_{i j}$, according to the Forster formalism. The critical distance $R$, above which the hopping frequency is dependent on the distance between polymer strands, has a dependence on both the distance separating the two charges of opposite sign in the singlet exciton, $R_{0}$, and the relative orientation of the dipole moments on both strands given by $R^{6}=R_{0}^{6} \times\left[\cos \left(\theta_{i j}\right)-3 \cos \left(\theta_{i}\right) \cos \left(\theta_{j}\right)\right]$, where $\theta_{i j}$ is the angle between the long axis of the strands $\mathrm{i}$ and $\mathrm{j}$ involved in the hopping process and $\theta_{i(j)}$ is the angle between the direction of the long axis of strand $\mathrm{i}(\mathrm{j})$ and the hopping direction. The third term is a thermal activated factor falling off exponentially with the energy barrier $\left(\Delta E_{i j}\right)$ for hopping which is given by difference between the exciton energy on the two conjugated segments obtained from the atomistic quantum molecular calculations.

During the hopping process, an exciton placed at the centre of a certain polymer strand only jumps for the neighbour strand which has the greatest hopping frequency. After each hopping event, there is a waiting time for all the excitons given by: 


$$
\tau=-\frac{\ln (X)}{w_{i j}}
$$

being $X$ a random number uniformly distributed between 0 and 1 .

On the first computer iteration it is established a queue of increasing time events. The event with the smallest time of occurrence takes place and is removed from the queue. This time of occurrence is then subtracted to the time of the other events and a new hopping event is inserted in the queue. When the event with the smallest waiting time corresponds to the lifetime of an exciton, the radiative decay of that exciton occurs being it removed from the queue. Using this strategy, based on the first reaction method $(\mathrm{FRM})^{11}$, we follow the time evolution of exciton diffusion and decay in our polymeric network.

\section{Results and discussion}

Since the diffusion of singlet intramolecular excitons will occur between polymer strands with and without similar lengths, we first calculate the energy barriers that a singlet exciton has to overcome within the built PPV network. Figure 1 shows the distribution of the strand lengths within polymer network and the energy barriers that a singlet exciton has to overcome to hop between a strand with the average length (7 monomers) and others with different length. Our results show that the energy barrier that an exciton has to overcome is higher for shorter polymer strands than for longer ones (see Figure 1). These results suggest that excitons tend to decay on longer strands, in agreement with experimental observations ${ }^{12}$.

Due to the architecture of an OSC, the cathode usually works like a mirror leading

to optical interference. As a result there is a non-uniform exciton generation inside the 
polymer-based layer due to light absorption which reaches a maximum value at the middle distance between the electrodes for a layer with $100 \mathrm{~nm}$ thickness ${ }^{13}$, assuming a uniform refraction index throughout the layer. Taking this into account, we have generated, within the 3D PPV networks, a Gaussian distribution of excitons along the direction perpendicular to the electrodes surface, with its mean value located at $50 \mathrm{~nm}$ from both electrodes, and uniform randomly distributed along the direction parallel to the electrodes surface.

Figure 2 shows the normalized photoluminescence due to a single pulse of excitons generated within the polymer network at the same time. Here we do not consider subsequent exciton formation as a result of re-absorption of spontaneous photon emission. The absence of nonradiative channels for exciton decay leads to a perfect fitting of the normalized photoluminescence to a mono-exponential, with a radiative lifetime of $1.09 \mathrm{~ns}$, which is in very good agreement with the value estimated from the experiments reported elsewhere for pristine PPV(1.20 ns $)^{14}$.

One of the main advantages of our model as compared to others already published in the literature is that explicitly it takes into account the morphology at nanometre scale, by considering the spatial disorder due to the arrangement of strands within the polymer network. Although the long molecular axis of each conjugated segment (chromophore) is parallel to the electrodes surface, the relative orientation of the strands conjugated plane is usually different as well as their length. As a result there is spatial and energetic disorder which influences exciton diffusion. Figure 3 shows the variation of exciton population in the polymer strands within the PPV network as a function of their length. These results show a decrease of exciton population for the shorter strands and an 
increase for the longer strands due to exciton migration from shorter towards longer strands, which is in agreement with the experimental results ${ }^{12}$ and it is a consequence of the molecular properties obtained from our atomistic calculations.

The orientational disorder of the polymer strands affects the preferential direction for exciton diffusion. In a polymer layer with a parallel morphology to the electrodes surface, the angle between the strands in the plane parallel to the electrodes favour exciton diffusion along that plane (in our model is the xy plane), being less favourable along the direction perpendicular to the electrodes surface (the direction along the $\mathrm{z}$ axis)

(see Figure 4). The calculated exciton diffusion length $\left(L=\sqrt{L_{x}^{2}+L_{y}^{2}+L_{z}^{2}}\right)$ of $4.312 \mathrm{~nm}$ is in very good agreement to the experimental one reported by Markov et al. ${ }^{15}$ for PPV derivatives $\left(L_{D}=5 \pm 1 \mathrm{~nm}\right)$.

Considering that $L_{D}=\sqrt{D \times \tau_{0}}$, where $D$ is the diffusion coefficient, $L_{D}$ is the diffusion length and $\tau_{0}$ is the exciton life-time, we can calculate the diffusion coefficient of excitons in the PPV network. A diffusion coefficient of $1.71 \times 10^{-4} \mathrm{~cm}^{2} / \mathrm{s}$ was obtained, which is similar to the value obtained experimentally $\left(2.0 \times 10^{-4} \mathrm{~cm}^{2} / \mathrm{s}^{14}\right)$.

\section{Conclusions}

In this work we have described a new computational model developed to simulate exciton diffusion within a 3D polymer network, which uses the results from atomistic calculations to describe all the relevant molecular properties and the arrangement of the polymer strands. From the model, exciton diffusion is found to depend on the energetic and spatial disorder. The results obtained for the exciton lifetime, diffusion length, and diffusion coefficient in PPV with a morphology parallel to the electrodes surface are in 
very good agreement with the experimental ones published in the literature for polymer layers deposited by spin coating.

In particular, it was shown that when the polymer strands have their long axes parallel to the electrodes surface the exciton diffusion occurs preferentially along the planes parallel to them. On the bases of this result we conclude that in bulk heterojunctions solar cells deposited by the spin-coating technique, the efficient interfaces for exciton dissociation will be the ones that are perpendicular to the electrodes surface. This study provides a deeper insight on the effects of energetic and spatial disorder on exciton diffusion in polymeric systems, as well as a novel concept for improving the efficiency of photovoltaic devices by adjusting the direction of donor/acceptor interface within a conjugated polymer active layer.

\section{Acknowledgement}

This work is part of the research projects POCTI/CTM/41574/2001 and CONCREEQ/443/EEI/2005, approved by the Portuguese Foundation for Science and Technology (FCT) and support by the European Community Fund FEDER. One of us (H.M.C.B.) is also indebted to FCT for financial support under $\mathrm{PhD}$ grant $\mathrm{N}^{\mathrm{o}}$ SFRH/BD/22143/2005 and Fundação Caloust Gulbenkien for supporting his travel expenses to present this work on NanoSMat 2008 conference. All the authors thank Professor M. I. C. Ferreira and Dr. Graham Hungerford for useful discussions. 


\section{List of figure captions:}

Figure 1 - Energy barrier height that an intramolecular exciton of PPV has to overcome to hop between a polymer strand of 7 monomers length to a strand with other length for PPV (a) and the distribution of the strand lengths within the polymer network (b).

Figure 2 - The calculated normalized photoluminescence (PL) within the PPV network as a function of time. The squares represent the number of photons emitted per time intervals of $0.2 \mathrm{~ns}$ due to exciton decay. The line represents the exponential fit to the calculated results.

Figure 3 - Fraction of excitons created (closed marks) within polymer network as well as fraction of excitons decay (open marks) in PPV, considering the strand within the polymer network.

Figure 4 - The exciton diffusion length (L) in the PPV network along the directions parallel and perpendicular to the electrodes surfaces. 
Hélder M C Barbosa, Helena M G Correia and Marta M D Ramos

Quantum and Nanoscale Modelling of Excitons Dynamics in Polymeric Systems

Figure 1
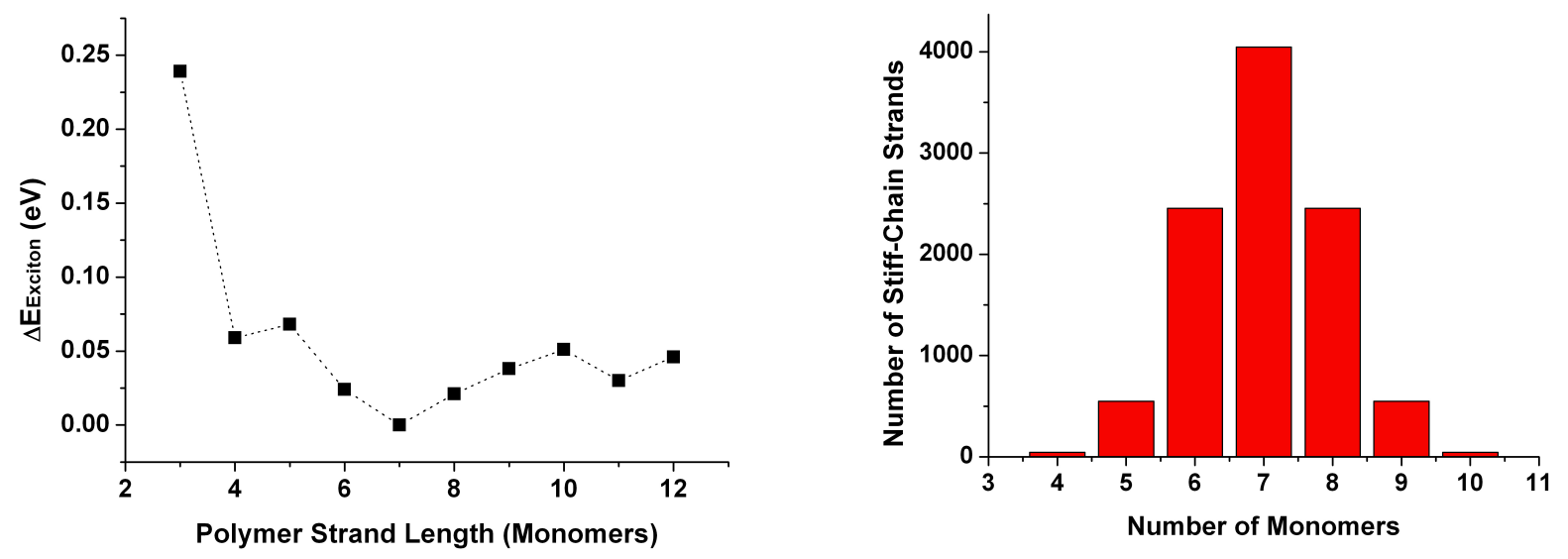

(a)

(b) 
Hélder M C Barbosa, Helena M G Correia and Marta M D Ramos

Quantum and Nanoscale Modelling of Excitons Dynamics in Polymeric Systems

Figure 2

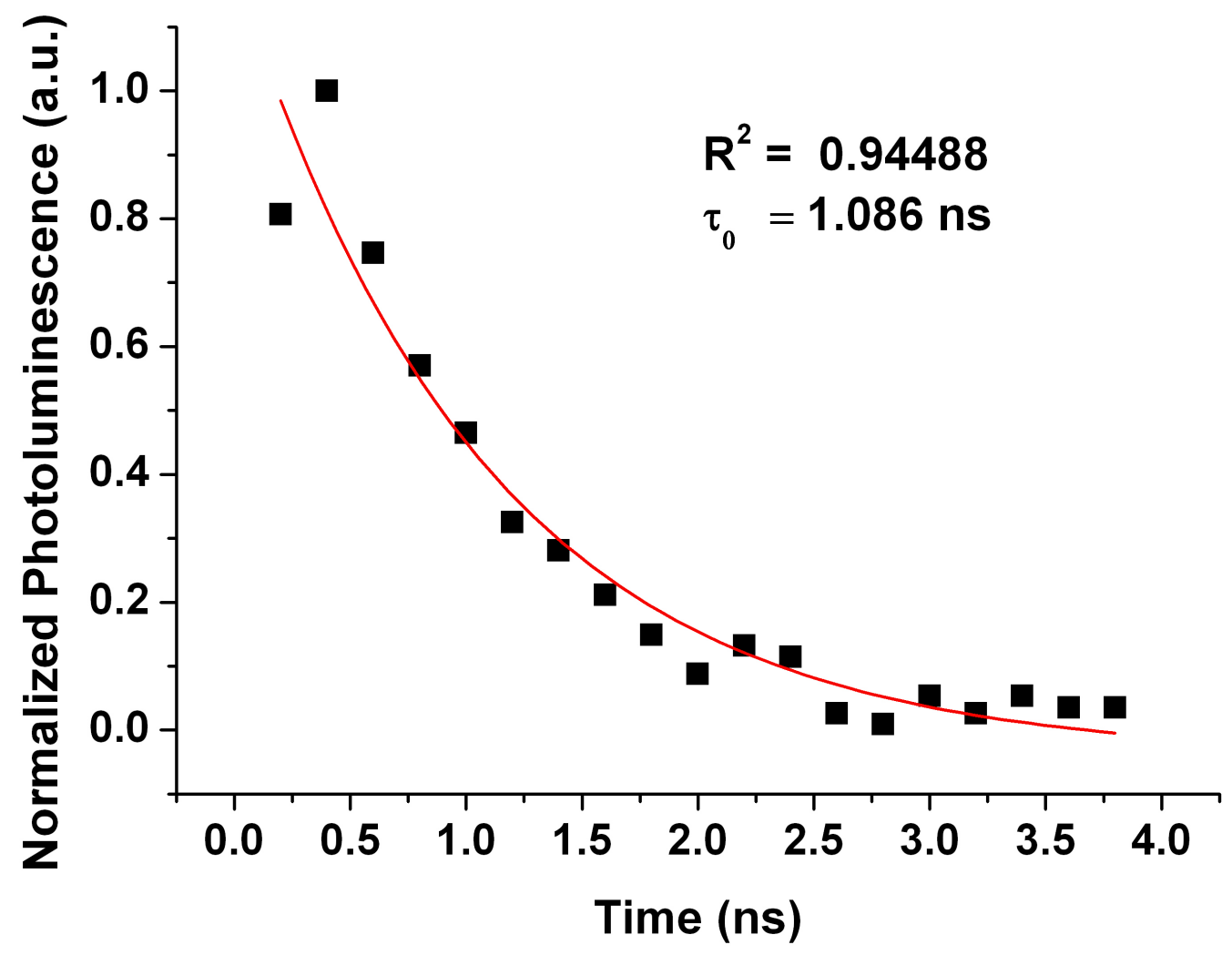


Hélder M C Barbosa, Helena M G Correia and Marta M D Ramos

Quantum and Nanoscale Modelling of Excitons Dynamics in Polymeric Systems

Figure 3

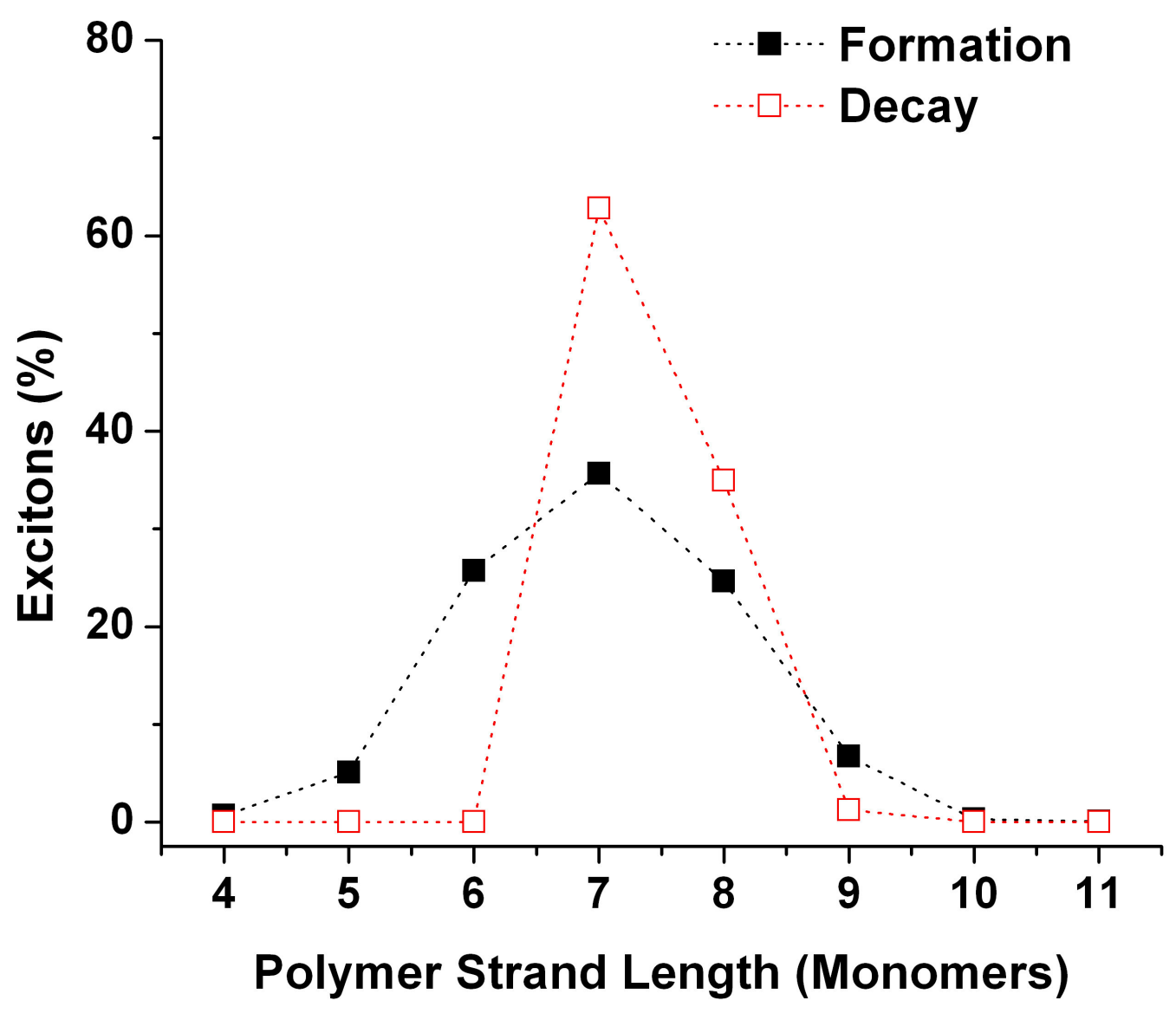


Hélder M C Barbosa, Helena M G Correia and Marta M D Ramos

Quantum and Nanoscale Modelling of Excitons Dynamics in Polymeric Systems

Figure 4

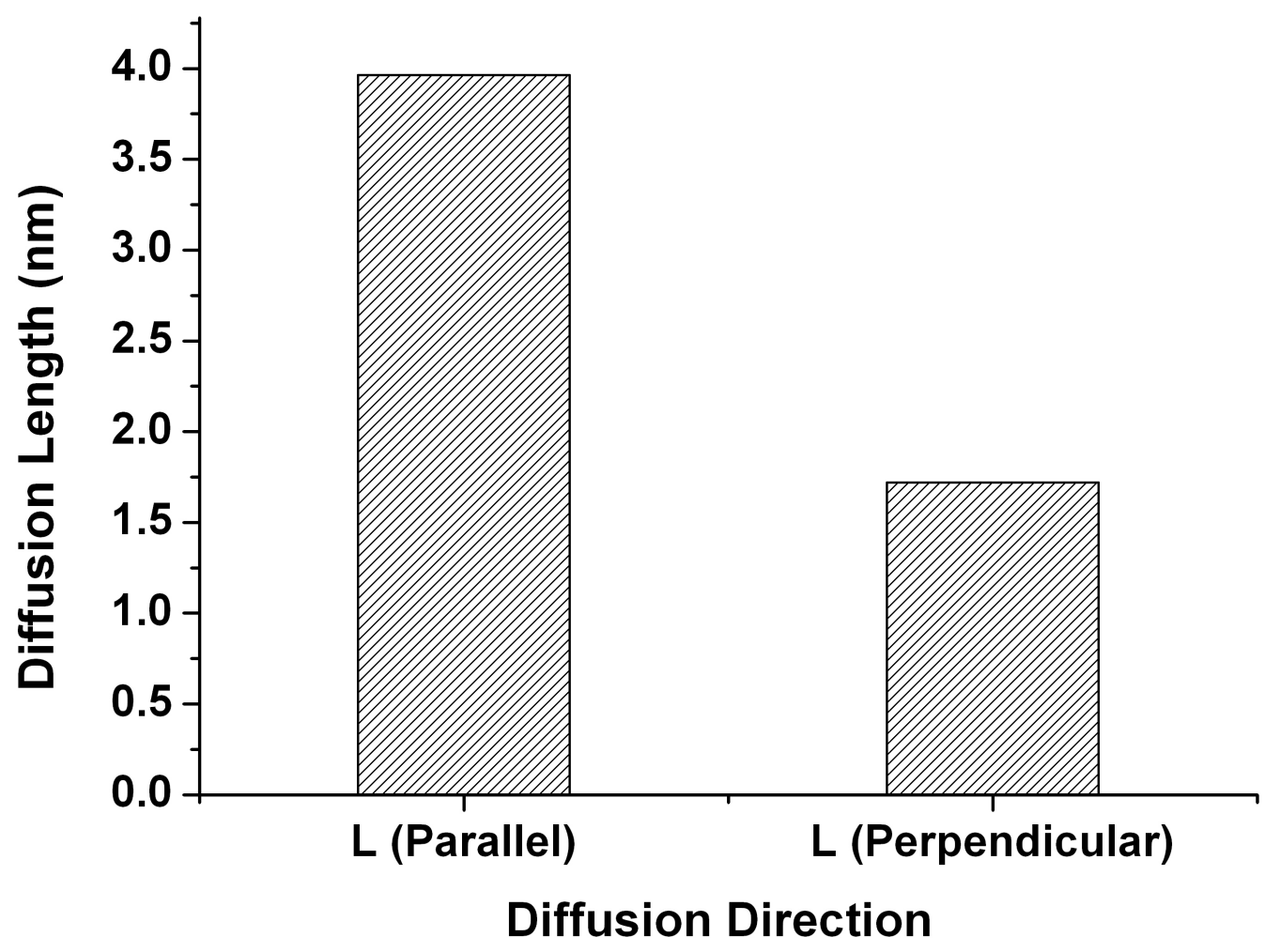




\section{References}

1. N.S. Sariciftci, L. Smilowitz, A.J. Heeger, F. Wudl, Science 258, 1474 (1992)

2. P. Peumans, A. Yakimov, S.R. Forrest, Journal of Applied Physics 93, 3693 (2003)

3. P.K. Watkins, A.B. Walker, G.L.B. Verschoor, Nano Lett. 5, 1814 (2005)

4. M.M.D. Ramos, H.M.G. Correia, Appl. Surf. Sci. 248, 450 (2005)

5. A.M. Stoneham, M.M.D. Ramos, A.M. Almeida, H.M.G. Correia, R.M. Ribeiro, H. Ness, A.J. Fisher, J. Phys.-Condes. Matter 14, 9877 (2002)

6. M. Scheidler, U. Lemmer, R. Kersting, S. Karg, W. Riess, B. Cleve, R.F. Mahrt, H. Kurz, H. Bassler, E.O. Gobel, P. Thomas, Phys. Rev. B 54, 5536 (1996)

7. T.S. Ahn, N. Wright, C.J. Bardeen, Chem. Phys. Lett. 446, 43 (2007)

8. C.Y. Yang, F. Hide, M.A. Diaz-Garcia, A.J. Heeger, Y. Cao, Polymer 39, 2299 (1998)

9. M.M.D. Ramos, H.M.G. Correia, J. Phys.: Condens. Matter 18, S429 (2006)

10. D.S. Wallace, A.M. Stoneham, W. Hayes, A.J. Fisher, A.H. Harker, J. Phys.Condes. Matter 3, 3879 (1991)

11. J.J. Lukkien, J.P.L. Segers, P.A.J. Hilbers, R.J. Gelten, A.P.J. Jansen, Physical Review E 58, 2598 (1998)

12. R. Kersting, U. Lemmer, R.F. Mahrt, K. Leo, H. Kurz, H. Bassler, E.O. Gobel, Phys. Rev. Lett. 70, 3820 (1993)

13. H. Hoppe, N. Arnold, N.S. Sariciftci, D. Meissner, Sol. Energy Mater. Sol. Cells $80,105(2003)$ 
14. M. Yan, L.J. Rothberg, F. Papadimitrakopoulos, M.E. Galvin, T.M. Miller, Phys. Rev. Lett. 73, 744 (1994)

15. D.E. Markov, E. Amsterdam, P.W.M. Blom, A.B. Sieval, J.C. Hummelen, J. Phys. Chem. A 109, 5266 (2005) 